\title{
FOXP3 is a novel transcriptional repressor for the breast cancer oncogene SKP2
}

\author{
Tao Zuo,, Runhua Liu, ${ }^{1}$ Huiming Zhang, ${ }^{1}$ Xing Chang, ${ }^{1}$ Yan Liu, ${ }^{1}$ Lizhong Wang, ${ }^{1}$ \\ Pan Zheng, 1,2,3 and Yang Liu1,3,4 \\ 1Department of Surgery, Section of General Surgery, Division of Immunotherapy, ${ }^{2}$ Department of Pathology,

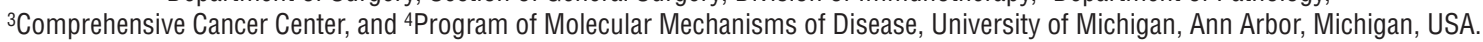

\begin{abstract}
S-phase kinase-associated protein 2 (SKP2) is a component of the E3 ubiquitin ligase SKP1-Cul1-Fbox complex. Overexpression of SKP2 results in cell cycle dysregulation and carcinogenesis; however, the genetic lesions that cause this upregulation are poorly understood. We recently demonstrated that forkhead box P3 (FOXP3) is an $\mathrm{X}$-linked breast cancer suppressor and an important repressor of the oncogene ERBB2/HER2. Since FOXP3 suppresses tumor growth regardless of whether the tumors overexpress ERBB2/HER2, additional FOXP3 targets may be involved in its tumor suppressor activity. Here, we show that mammary carcinomas from mice heterozygous for a Foxp 3 mutation exhibited increased $S k p 2$ expression. Ectopic expression of FOXP3 in mouse mammary cancer cells repressed SKP2 expression with a corresponding increase in p27 and polyploidy. Conversely, siRNA silencing of the FOXP3 gene in human mammary epithelial cells increased SKP2 expression. We also show that Foxp3 directly interacted with and repressed the $S k p 2$ promoter. Moreover, the analysis of over 200 primary breast cancer samples revealed an inverse correlation between FOXP3 and SKP2 levels. Finally, we demonstrated that downregulation of SKP2 was critical for FOXP3-mediated growth inhibition in breast cancer cells that do not overexpress ERBB2/HER2. Our data provide genetic, biochemical, and functional evidence that FOXP3 is a novel transcriptional repressor for the oncogene SKP2.
\end{abstract}

\section{Introduction}

Cancer pathogenesis involves both the inactivation of tumor suppressor genes and the activation of oncogenes $(1,2)$. One of the most fascinating aspects of cancer biology is the interaction between cancer suppressor genes and oncogenes. Most of these interactions are at posttranslational levels. For instance, proteins encoded by tumor suppressor genes can inactivate oncogenes. One of the most clearly studied cases is tumor suppressor Rb, which inhibits the $E 2 F$ family members of oncogenes $(1,2)$. Conversely, oncogenes can overcome the tumor suppressor proteins. For example, S-phase kinase-associated protein 2 (SKP2) causes the degradation of tumor suppressor FOXO (3) as well as CDK inhibitors, such as p27 $(4,5)$. It is less clear whether such antagonism exists at the transcriptional level. However, a recent study suggests that FOXO may repress the expression of cyclin $D(6)$, a well-known oncogene. Likewise, we have recently demonstrated that forkhead box P3 (FOXP3), an X-linked tumor suppressor, represses transcription of ERBB2/HER2 oncogene (7).

The high-level expression of SKP2 was reported in a significant proportion of cancers (5). SKP2 is a component of the E3 ubiquitin ligase SCF with specificity for CDK inhibitor p27. However, under physiological conditions, Skp2 expression is maximal at the $\mathrm{S}$ and G2 phases and appears to primarily mediate p27 degradation at the G2 $(8,9)$ but not G0 and G1 phases (10). As such, Skp2 was found to be essential for progression into mitosis in cell cycles $(4,9,11)$. Targeted mutation of Skp2 causes delayed animal growth and cellular polyploidy, which were prevented by an additional

Nonstandard abbreviations used: ChIP, chromatin immunoprecipitation; FOXP3, forkhead box P3; HMEC, human mammary epithelial cell; SKP2, S-phase kinase-associated protein 2 .

Conflict of interest: The authors have declared that no conflict of interest exists. Citation for this article: J. Clin. Invest. 117:3765-3773 (2007). doi:10.1172/JCI32538. deletion of $p 27^{-/-}$mice $(8,9)$. The increased expression of SKP2 has been shown in nearly $50 \%$ of breast cancers, especially among those with early onset and poor prognosis (12-15). The oncogenic effect of Skp2 overexpression is substantiated by cancer development in Skp2 transgenic mice $(16,17)$. However, genetic lesions responsible for SKP2 overexpression in cancers remain largely undefined although gene amplification of SKP2 has recently been shown in non-small cell lung carcinomas (18). The transcriptional regulation of SKP2 has also been studied recently $(19,20)$, which paves the way to study whether aberrant genetic alterations on some specific transcriptional regulators of SKP2 could cause overexpression of this oncogene in cancers.

FOXP3 is a new member of the Forkhead/winged helix family of the transcription factors and was identified by position cloning as the causative mutation for lethal autoimmune diseases (21-24). In the $\mathrm{T}$ cell lineage, mutation of the Foxp3 gene resulted in ablation of the suppressor function of Tregs although it is still debatable whether such mutation ablates the Treg lineages (25), as was originally proposed $(26,27)$. In addition, we reported that Foxp 3 mutation in nonhematopoietic cells, most likely the thymic epithelial cells, is necessary and sufficient to cause abnormal thymopoiesis (28). In support of an epithelial intrinsic function of Foxp3, we have recently observed that a germline mutation of Foxp3 resulted in a high rate of spontaneous breast cancer and increased susceptibility to carcinogens in the mouse (7). In addition, widespread deletion and somatic mutations of FOXP3 have also been observed in human breast cancer samples. While we have identified FOXP3 as a transcriptional repressor of the HER2/ERBB2 oncogene (7), FOXP3 also suppressed growth and induced the cell death of MCF-7, a breast cancer line without HER2/ERBB2 overexpression (7). Therefore, it is likely that Foxp3 may affect other pathways involved in breast cancer. Given the role of Skp2 in breast cancer (12-15), we determined whether Foxp3 may regulate its expression 
A

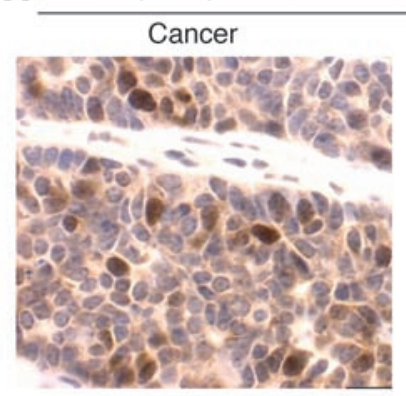

Skp2 expression in mouse mammory tissue

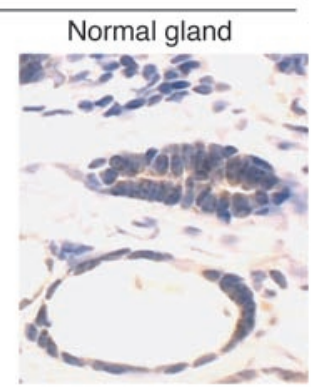

B

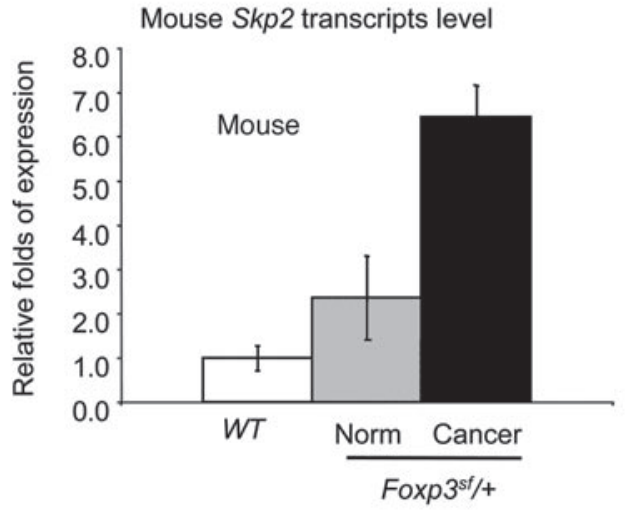

in normal and malignant breast epithelial cells. Here, we report that Foxp3 is a transcriptional repressor of $S k p 2$, a breast cancer oncogene. These data indicate that defect in FOXP3 is a potential mechanism for SKP2 upregulation in breast cancer and illustrate an intriguing interplay between the oncogenes and tumor suppressor genes in cell-cycle regulation and carcinogenesis.

\section{Results}

Inactivation of the Foxp 3 locus resulted in increased Skp2 expression. We have reported that mice heterozygous for the Foxp 3 gene developed spontaneous mammary cancer at high rates (7). Moreover, while most of the Foxp3 transcripts in the normal epithelial cells were from WT alleles, all of the transcripts from the cancerous tissues were transcribed from the mutant allele. Thus, in the cancer cells, the Foxp 3 locus is silenced. To determine whether Foxp 3 represses Skp2 expression, we stained the normal and cancerous mammary tissues with anti-Skp2 and anti-p27 antibodies. As shown in Figure $1 \mathrm{~A}, \mathrm{Skp} 2$ was found to be highly expressed in cancer cells but not in normal epithelial cells from the same mouse. To quantify increases in $S k p 2$ transcripts, we isolated cells from frozen sections by laser microdissection and extracted mRNA for real-time RT-PCR analysis. We compared the expression of Skp2 in normal mammary epithelial cells from either WT or Foxp $3^{f /+}$ mice as well as mammary cancer tissues from mutant mice. As shown in Figure $1 \mathrm{~B}$, in comparison with the WT epithelial cells, the heterozygous epithelial cells expressed 2-fold higher levels of Skp2, which suggests a Foxp3 gene dose effect on the levels of Skp2. Moreover, in the cancerous tissue that has silenced the WT allele (7), expression of Skp2 was substantially enhanced.

A potential caveat of this interpretation is that upregulation of SKP2 may be due to cancer rather than to the silencing of the Foxp3 locus. Although the WT mice had lower incidences and later onsets of mammary cancer than the heterozygous mice, cancer did

\section{Figure 1}

A naturally occurring mutation in the Foxp3 locus results in increased Skp2 expression. (A) Immunohistochemical staining of the Skp2 protein in mammary cancers and an adjacent normal mammary gland in 1 Foxp $3^{\text {st/ }}$ mouse. Original magnification, $\times 40$. (B) Relative levels of Skp2 transcripts in normal mammary epithelium of WT and Foxp3 $3^{\text {sft+ }}$ mice and the cancerous tissue in the Foxp $3^{\text {st/ }}$ mice, as revealed by real-time RT-PCR of LCM samples. The expression of Skp2 was normalized against the internal control, the Hprt gene. Data shown are means \pm SD of 3 samples in each group. Highly significant differences were observed between cancerous and normal tissue $(P<0.001$, ANOVA test when either internal standards were used).

arise, both spontaneously and in response to carcinogen treatment (7). Thus, by comparing mouse mammary cancer tissues from WT and Foxp $3^{s f /+}$ mice for expression of Skp2, one may be able to discern the contribution of Foxp3 mutation versus the nonspecific effect of cancer growth. As shown in Table 1, 80\% of the spontaneous cancers in the WT mice did not overexpress Skp2. In contrast, $71 \%$ of the spontaneous tumors from the Foxp $3^{\text {sf/+ }}$ mice did. A similar trend was observed in the carcinogen-induced mammary tumors. Thus, inactivation of the Foxp3 locus is likely responsible for increased Skp2 expression in the mammary tumors.

Foxp3 as a transcriptional repressor of Skp2. Since Foxp3 is a transcription factor capable of repressing or promoting the expression of a large cohort of genes $(29,30)$, we evaluated whether Skp2 can be a direct target of Foxp3. We first transfected a mouse mammary cancer line, TSA, with the V5-tagged Foxp3 protein and generated a polyclonal Foxp3-V5, CL30, and 2 subclones, CL302 and CL305. Using real-time PCR analysis, we found that the CL302 and 305 have approximately 5-fold higher Foxp 3 transcript than the CL30 line. Skp2 transcripts were found to decrease by around 10- to 20fold in the Foxp3-V5 transfectant line or clones compared with the vector control. The extent of reduction correlated with the Foxp3 transcript levels. In contrast, no changes in $p 27 \mathrm{mRNA}$ levels were detected (Figure 2A). Since Skp2 regulates the degradation of $\mathrm{p} 27$, we also examined the levels of these 2 proteins in Foxp3-V5 transfectants. As shown in Figure 2B, Foxp3 transfection dramatically reduced Skp2. Correspondingly, p27 was significantly increased in the Foxp3-V5 transfectant. To determine whether the increase of $\mathrm{p} 27$ was caused by more rapid degradation, we treated vector

\section{Table 1}

Inactivation of the Foxp3 locus contributed to Skp2 elevation in mouse mammary tumors

\begin{tabular}{|c|c|c|c|c|}
\hline & \multicolumn{2}{|c|}{ Spontaneous tumor } & \multicolumn{2}{|c|}{ Carcinogen-induced tumor } \\
\hline & $\begin{array}{c}\text { WT } \\
(n=5)\end{array}$ & $\begin{array}{c}\text { Foxp3 }^{\mathrm{s} / /+} \\
(n=7)\end{array}$ & $\begin{array}{c}\text { WT } \\
(n=10)\end{array}$ & $\begin{array}{c}\text { Foxp3 } 3 \text { st/ } \\
(n=12)\end{array}$ \\
\hline \multicolumn{5}{|c|}{ Skp2 } \\
\hline$(-)$ & 4 & 2 & 7 & 4 \\
\hline$(+)$ & 1 & 5 & 3 & 8 \\
\hline
\end{tabular}

Arabic numerals indicate the number of mice carrying mammary tumors. A7,12-Dimethylbenz[a]anthracene was used to induce mammary tumorigenesis in mice. Detailed protocol has been described previously (7). $\chi^{2}$ test revealed that the difference in frequency of SKP+ cases between the tumors (including both spontaneous and chemically induced) from the Foxp3 $3^{\text {sf/+ }}$ mice and the WT littermates was significant $(P=0.0152)$. 
A

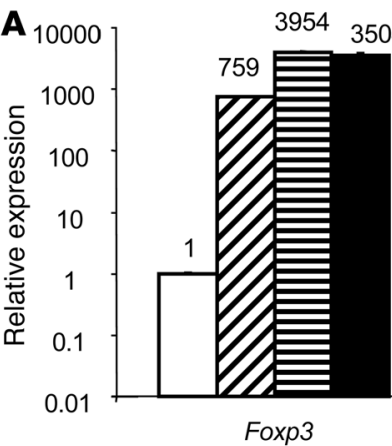

C 
Foxp3$$
\text { Vector }
$$

TSA-Vector

TSA-Foxp3V5 CL30

TSA-Foxp3V5 CL302

TSA-Foxp3V5 CL305
B

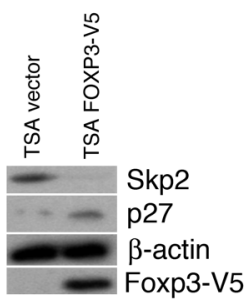

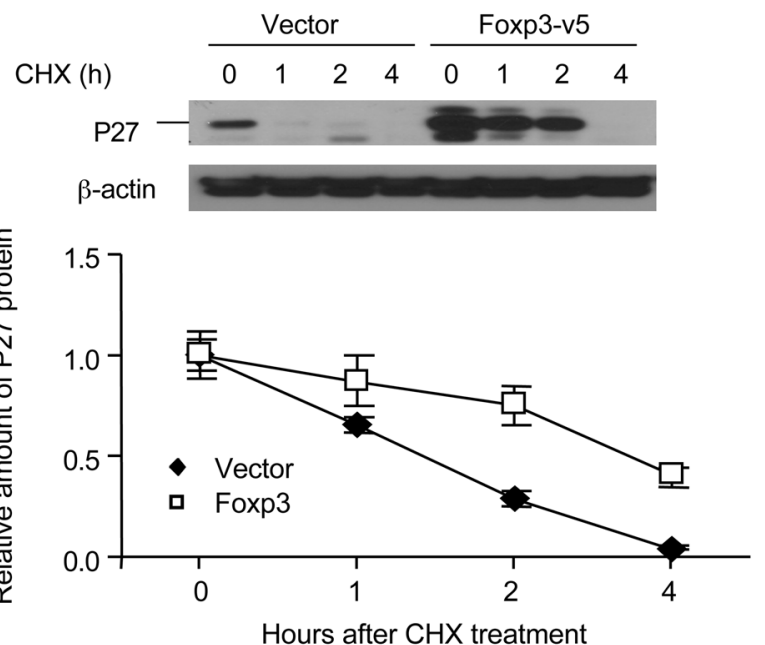

D
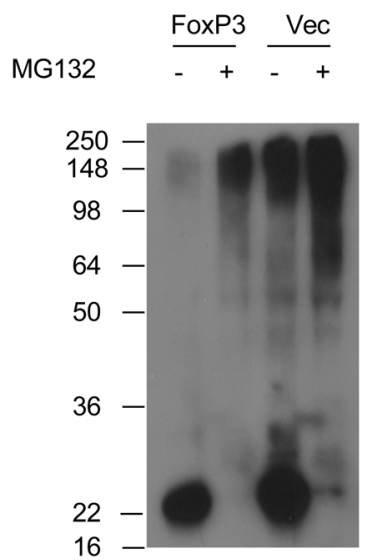

IP: anti-p27, WB, anti-polyUb

\section{Figure 2}

Foxp3 represses Skp2 transcription. (A) Transfection of Foxp3-V5 into TSA cells repressed expression of the Skp2 gene. The mRNA levels of Foxp3, Skp2, and p27 were measured by real-time PCR for the vector control cells, polyclonal Foxp3-V5 transfectants (CL30), and 2 stable Foxp3-V5 transfectant clones, CL302 and CL305. Data shown are relative amounts of transcripts after normalizing against the amounts of total RNA based on the levels of Hprt mRNA. The means of the vector group are artificially defined as 1.0. Data shown are means \pm SD of 3 independent experiments. (B) Foxp3 reduces Skp2 with a corresponding increase in p27. Lysates of Foxp3-V5 or vector-transfected TSA cell lines were analyzed by Western blot using SKP2, p27, $\beta$-actin, and anti-V5 (which recognize V5-tagged Foxp3) antibodies. (C) Foxp3 increased stability of p27. Vector or Foxp3-V5 transfectants were treated with cycloheximide $(\mathrm{CHX}, 100 \mu \mathrm{M})$ for the indicated intervals. Cells were collected and p27 protein levels were detected by Western blot. The upper panel shows representative experiments while the lower panel shows the decay of p27, using time 0 as 1.0. Protein loading equivalence was assessed by the expression of $\beta$-actin. The relative intensity of bands was measured relative to their respective $\beta$-actin bands using BandScan software (version 4.3; Glyco). (D) Polyubiquitination of the p27 in vector or Foxp3 transfectants. Vector or Foxp3-V5-transfected TSA cell lines were either left untreated or treated with proteasome inhibitor MG132 $(40 \mu \mathrm{M})$ for 3 hours. Equal aliquot of the cellular lysates were immunoprecipitated with anti-p27 antibodies. The precipitates were separated by SDS-PAGE and transferred into nitrocellulose membrane, which were then autoclaved in water for 30 minutes and blotted with HRP-conjugated anti-polyubiquitin antibody Ub P4D1 (33).

or Foxp3-V5-transfected TSA cells with cycloheximide and measured the levels of p27 at 0,1,2, and 4 hours after treatment by Western blot. As shown in Figure 2C, p27 was degraded at a much faster rate in the vector-transfected TSA cells. Consistent with this notion, we observed reduced ubiquitination of p27 in the Foxp3transfected cells (Figure 2D).

To further confirm that the downregulation of Skp2 by Foxp3 occurred at the transcription level, we cloned the $2.0-\mathrm{kb}$ region upstream of the murine Skp2 gene into the luciferase reporter vector pGL2 and tested the effects of Foxp3 of this promoter's activity by luciferase assay. As shown in Figure 3A, Foxp3 substantially repressed the promoter activity of the Skp2 gene.

Analysis of the Skp2 promoter revealed 4 potential binding sites within the $2 \mathrm{~kb}$ promoter region (Figure $3 \mathrm{~B}$ ). We carried out chromatin immunoprecipitation (ChIP) to determine whether Foxp3 binds to the promoter. The nuclear preparations from the Foxp3-transfected cells were fixed with paraformaldehyde. After sonication, the Foxp3-associated genomic DNA was immunoprecipitated and quantitated by real-time PCR. To avoid artifacts associated with differential amplification, we compared the quantity of precipitated DNA with the total input of genomic DNA, amplified by the same pairs of primers. In addition, the small amount of DNA precipitated by the IgG control was subtracted. As shown in Figure 3B, the primers corresponding to the $-0.8-\mathrm{kb}$ and $-1.2-\mathrm{kb}$ regions yielded significant amounts of product, which was equal to $5 \%-6 \%$ of input DNA. In contrast, those corresponding to either the -2.2 - or $+0.6-\mathrm{kb}$ region yielded no specific signal. 


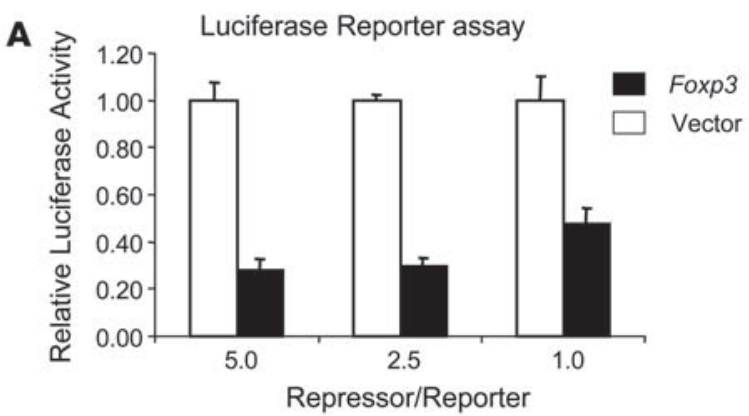

B Forkhead domain DNA binding core motif :

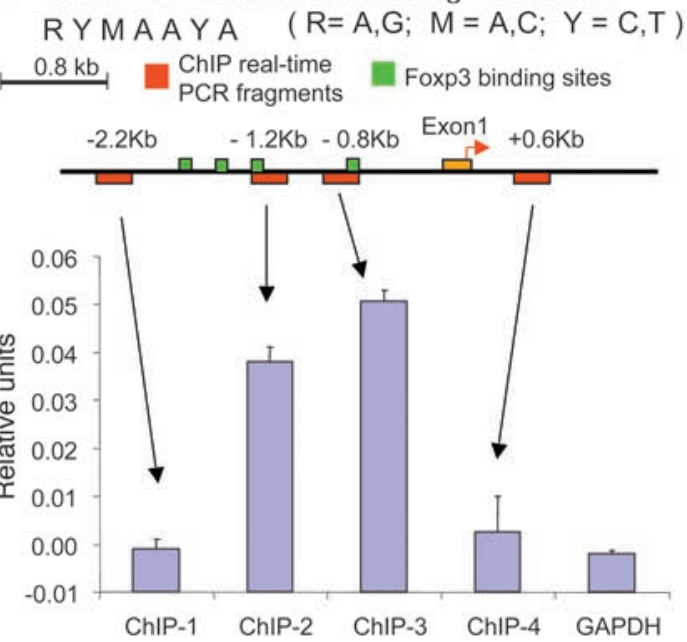

C Site-directed mutagenesis Forkhead binding sites in mouse Skp2 promoter

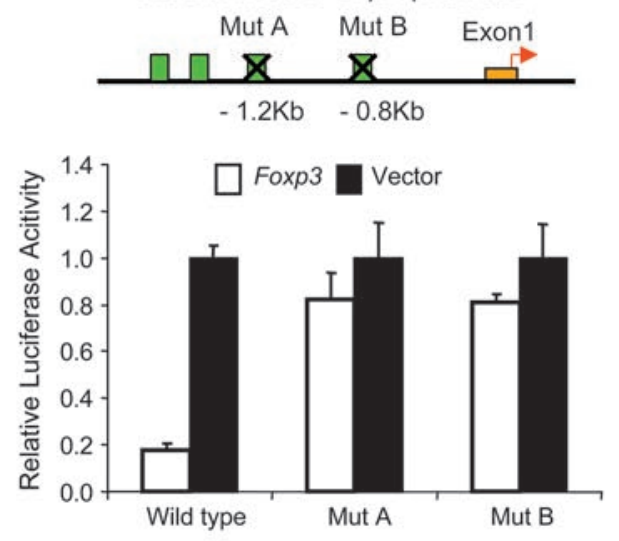

To determine the significance of the interaction, we determined whether deletion of either binding site disrupted the repression of promoter activity by Foxp3. As shown in Figure 3C, while the WT promoter was repressed by Foxp3, deletion of either site eliminated the repression. Thus, data presented in this section demonstrate that the binding of Foxp 3 to specific sites in the Skp2 promoter is essential for Foxp3 repression of Skp2 expression.

Foxp3 expression caused polyploidy of breast cancer cell lines. One of the clearly defined functions of Skp2 is to regulate the p27 levels at the $\mathrm{G} 2 / \mathrm{M}$ phase in the cell cycle $(4,9)$. As a manifestation of defective G2/M transition, liver cells in the Skp2-deficient mice showed drastically increased polyploidy $(8,9)$. Therefore, the percentage of cells with polyploidy can be used as a valuable parameter for Skp2

\section{Figure 3}

Foxp3 binding to $S k p 2$ is important for transcriptional repression. (A) Foxp3 represses mouse Skp2 promoter activity. Either Foxp3 cDNA or empty vector was transiently cotransfected with reporter vector at different ratios illustrated in the figure. Cells were transfected with either vector control or Foxp3 $(1 \mu \mathrm{g} /$ well $)$ in conjunction with the luciferase reporter driven by $5^{\prime}$ promoter regions of the Skp2 gene $(0.2 \mu \mathrm{g}, 0.4$ $\mu \mathrm{g}$ and $1.0 \mu \mathrm{g}$ per well). Forty-eight hours later, the cell lysates were harvested and measured for luciferase activity. The luciferase activity from the cells transfected with the pGL2-basic vector was arbitrarily defined as 1.0. Data shown are means \pm SD of triplicates and have been repeated at least 3 times. (B) The upper panel depicts the $5^{\prime}$ region of the Skp2 gene. The lower panel shows the amount of DNA precipitated by anti-V $5 \mathrm{mAbs}$ after subtracting a minute portion precipitated by IgG control. The data shown are a fraction of the total genomic DNA isolated from the same number of cells. (C) Deletion of 1 of the 2 Foxp3-binding sites in the Skp2 promoter region prevented FOXP3mediated suppression. The deleted sequences are mut A: ACTAAACCAATATTCTAAT and mut B: TAAAAATAAACCATC. The promoter activity was measured in the human breast cancer line T47D.

function. We chose a Foxp3-transfected TSA cell line with moderate levels of the Foxp3-V5 protein to test the effect of Foxp3 expression (Figure 4A) on the cellular function of Skp2 in order to avoid possible artifacts associated with overexpression. Real-time PCR revealed that the levels of Foxp3 transcripts in the stable transfectants is about 4.5-fold that of the ex vivo mammary epithelial isolates after normalizing against Ck19 transcripts (Figure 4A). Since not all mammary epithelial cells express Foxp3, the difference between the transfectants and physiological levels of normal cells is likely to be even smaller. As shown in Figure 4B, only slightly more than $50 \%$ of the transfectants had demonstrable levels of the Foxp3-V5 fusion protein. This allowed us to compare the DNA contents of the Foxp $3^{\text {hi }}$ and Foxp $3^{\text {lo }}$ subsets from the same culture in addition to comparing them with control vector transfectants. As shown in Figure $4 \mathrm{~B}$, less than $1 \%$ of the control vector-transfected cells had $>4 \mathrm{C}$ DNA content, as expected. The same pattern was observed in the Foxp $3^{\text {lo }}$ subset from the Foxp3 transfectants. In contrast, about $25 \%$ of the Foxp $3^{\text {hi }}$ cells had $>4$ C DNA contents. To determine whether the polyploidy can be attributed to downregulation of Skp2, we ectopically expressed the Skp2 cDNA in the Foxp3-V5 transfects. As shown in Figure 4C, the ectopic expression of Skp2 significantly reduced the percentage of cells with polyploidy. These data demonstrate that, by suppressing Skp2 expression, Foxp3 has a very significant impact on cell cycle progression.

Foxp3 and SKP2 expression in normal and malignant buman breast epithelial cells. A critical issue is whether Foxp3 expression regulates SKP2 in human breast epithelial cells. To substantiate that inactivation of FOXP3 is a primary event leading to overexpression of SKP2, we transduced the early passage of normal human mammary epithelial cells (HMECs) with lentiviral vector encoding siRNA specific for FOXP3 or control lentiviral vector. The untransduced cells were eliminated by blasticidin. As shown in Figure 5A, the FOXP3 siRNA transduction caused a more than 100-fold reduction in the FOXP3 transcript. Corresponding to this, a 4-fold increase of the SKP2 transcripts was observed (Figure 5B). These data demonstrate that in HMECs, FOXP3 is an important regulator for the SKP2 gene.

To identify FOXP3 targets in malignant breast epithelial cells, we produced cell lines with the inducible expression of FOXP3 from MCF-7, a human mammary cancer cell line that does not overexpress the HER2 oncogene, as diagrammed in Figure 6, upper 

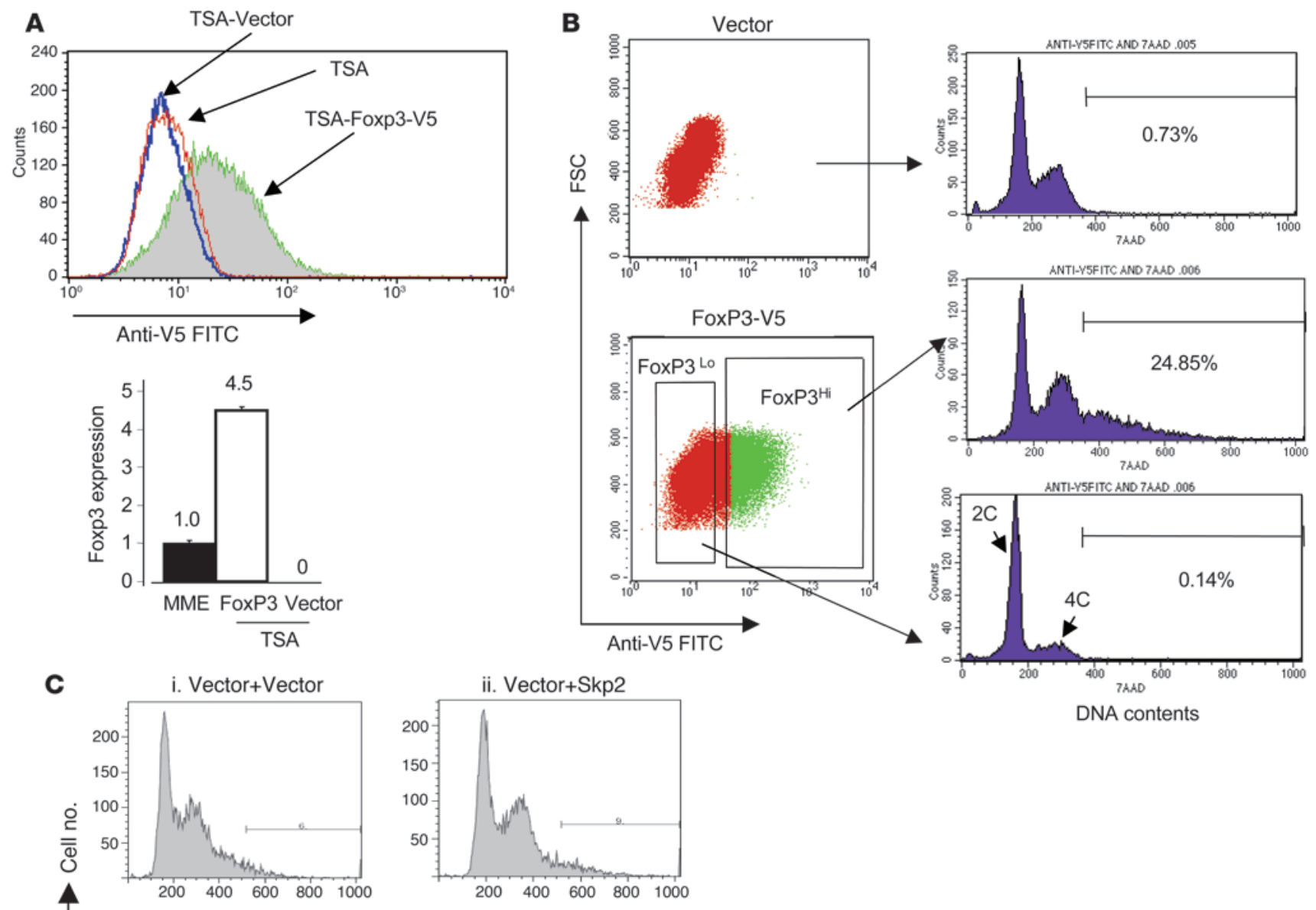

DNA contents
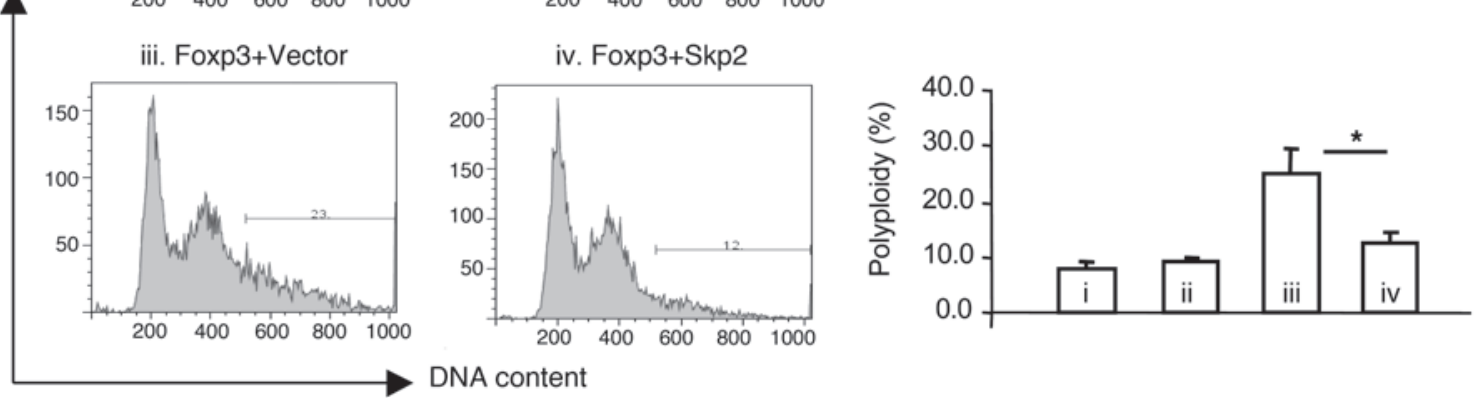

Figure 4

Polyploidy of the Foxp3 ${ }^{\text {hi }}$ but not the Foxp3 $3^{\text {lo }}$ mouse mammary cancer cells. (A) Modest Foxp3-V5 expression in the Foxp3 transfectant compared with either untransfected parental cells or control vector-transfected cells. Data shown in the upper panel are histograms depicting Foxp3-V5 protein levels, while those in the lower panel show levels of FoxP3 mRNA in Foxp3-V5- or vecotr-transfected TSA or LCM captured mouse mammary epithelial cells (MME), as measured by quantitative real-time PCR. (B) Foxp3 expression caused polyploidy. Left panel shows the level of the Foxp3-V5 fusion protein in vector control (top) or Foxp3-transfected TSA cells (bottom) while the right panel shows the DNA contents of vector control (top), Foxp3 ${ }^{\text {hi }}$ (middle), and Foxp3 ${ }^{\text {lo }}$ cells (bottom). (C) Ectopic expression of Skp2 alleviated polyploidy induced by Foxp3. Vector (i, ii) or Foxp3 (iii, iv) transfectants of TSA were transfected with either vector control or Skp2. After removing untransfected cells with blasticidin, the transfectants were fixed with Cytofix/Cytoperm buffer (BD) and tested with DNA contents using 7-AAD. Representative profiles are shown in the left and middle panels while summary data from 3 independent experiments are shown in the right panel. ${ }^{*} P<0.05$.

panel. We analyzed the expression of SKP2 at different time points after the cells were cultured in the absence of deoxycycline, which induced the expression of FOXP3 (7). The levels of SKP2 were quantitated by real-time PCR and were compared with control cell lines expressing GFP but not FOXP3 under the same conditions. The relative levels of the SKP2 transcripts of the control cell lines and the FOXP3-expressing cells at different times are presented in
Figure 6, lower panel. Using the levels of uninduced cells as references, we observed nearly a 4-fold reduction of SKP2 mRNA within 24 hours of removing deoxycycline in the FOXP3 transfectants. By 48 hours, more than an 8 -fold reduction was observed. No reduction of SKP2 transcript was observed in control cell lines cultured under the same conditions. These data demonstrate a rapid repression of the SKP2 transcripts following FOXP3 induction. 


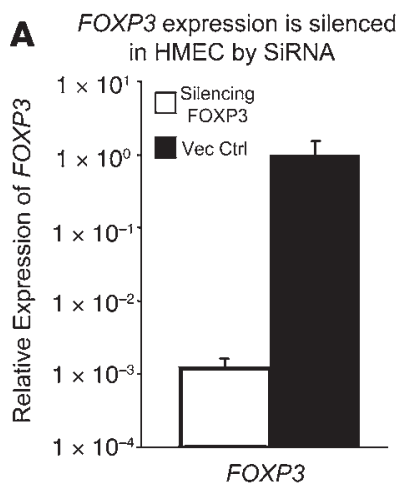

B

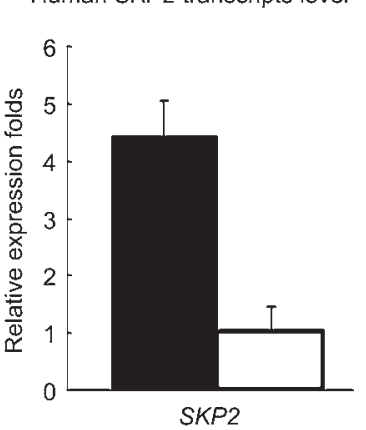

\section{Figure 5}

Silencing of FOXP3 resulted in upregulation of $S K P 2$ in primary HMEC. FOXP3 was silenced in HMEC by using siRNA. FOXP3 and $S K P 2$ transcripts were quantified by real-time PCR. The RNA inputs were normalized against housekeeping gene GAPDH. Data shown are means \pm SEM of relative levels of FOXP3 and represent 3 independent experiments ( $P<0.01$, Student's $t$ test). In $\mathbf{A}$, the vector control was defined as 1.0; in B, silenced cells were defined as 1.0. Data shown are means \pm SD of triplicates and represent 3 independent experiments.
We have reported that the FOXP3 locus is frequently inactivated in the majority of, although not all, mammary cancer tissues in humans. On the other hand, SKP2 is overexpressed in nearly $50 \%$ of the breast cancer samples (12-15). If a loss of FOXP3 contributes to SKP2 expression, one may expect an increased rate of the $\mathrm{SKP}^{+}$samples among the FOXP3- tumors. To address this issue, we independently stained and scored in a double-blinded fashion 206 cases of breast cancer samples in tissue microarray for their expression of SKP2 and FOXP3. As shown in Figure 7, among the FOXP3 ${ }^{+}$samples, less than $30 \%$ of the cells expressed SKP2. In contrast, more than $56 \%$ of the FOXP3- samples showed SKP2 overexpression. Statistical analysis revealed that the difference was highly significant $(P=0.0016)$.

The ectopic expression of SKP2 bypass FOXP3-mediated growth inbibition for a HER $2^{\text {lo }}$ breast cancer cell line. Our previous studies demonstrated that FOXP3 can suppress the growth of both ERBB2 ${ }^{\text {hi }}$ and ERBB2 ${ }^{\text {lo }}$ tumor cell lines. While the repression of ERBB2 $^{\text {hi }}$ tumor cell line TSA can be rescued by the ectopic expression of ERBB2, the target responsible for growth inhibition of the ERBB2 ${ }^{\text {lo }}$ tumor cells remained to be identified. To determine the relevance of SKP2 repression in growth inhibition by FOXP3, we ectopically expressed either vector or SKP2 into the MCF-7 cell line with TetOff inducible expression of FOXP3. The impact of the SKP2 expression was visualized by colony formation following TetOff induction of FOXP3. As shown in Figure 8, in the vector-transfected group, TetOff induction of FOXP3 wiped out all MCF-7 colonies, as expected. Remarkably, ectopic expression of SKP2 resulted in almost complete restoration of the colonies (Figure 8, lower panel) although the colony size is still somewhat less than in the culture without FOXP3 induction (Figure 8, upper and middle panels). These results demonstrate a critical role of SKP2 downregulation in the ERBB2 ${ }^{\text {lo }}$ breast cancer cell line.

\section{Discussion}

As a component of the SCF E3 complex, SKP2 has emerged as an important oncogenic protein for several major types of

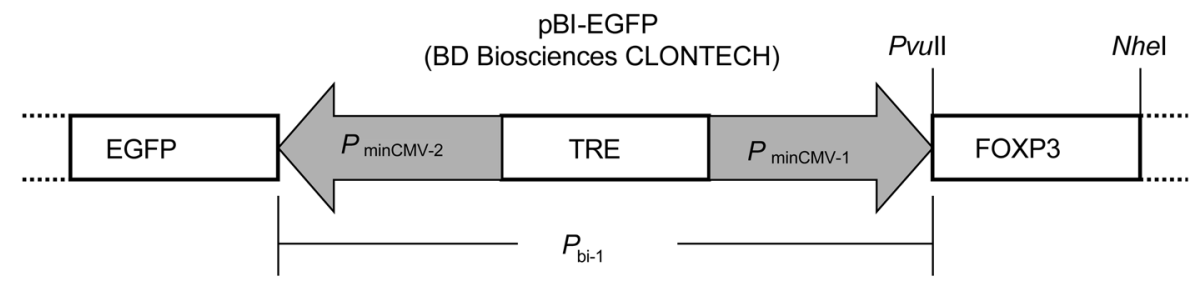

cancer, especially breast cancer (5). Overexpression of SKP2 is associated with a poor prognosis in breast cancer and is prevalent in cancer that develops in young women (15). While overexpression of Skp2 has been reported in multiple lineages of cancer and may confer resistance to antisteroid therapy $(5,15)$, the mechanisms for its overexpression have not been well understood. Here, we demonstrate that Foxp3, which we have recently demonstrated to be the first X-linked breast cancer suppressor gene, to our knowledge, is, we believe, a novel transacting regulator for Skp2 expression. Our conclusion is based on 4 lines of evidence.

First, inactivation of the Foxp3 locus resulted in the elevation of Skp2 expression. Mammary epithelial cells from mice heterozygous for Foxp3 mutation had 2-fold higher levels of Skp2. Since Foxp3 is an X-linked gene and is dose compensated (27), the elevation was likely due to Skp2 overexpression among the cells 


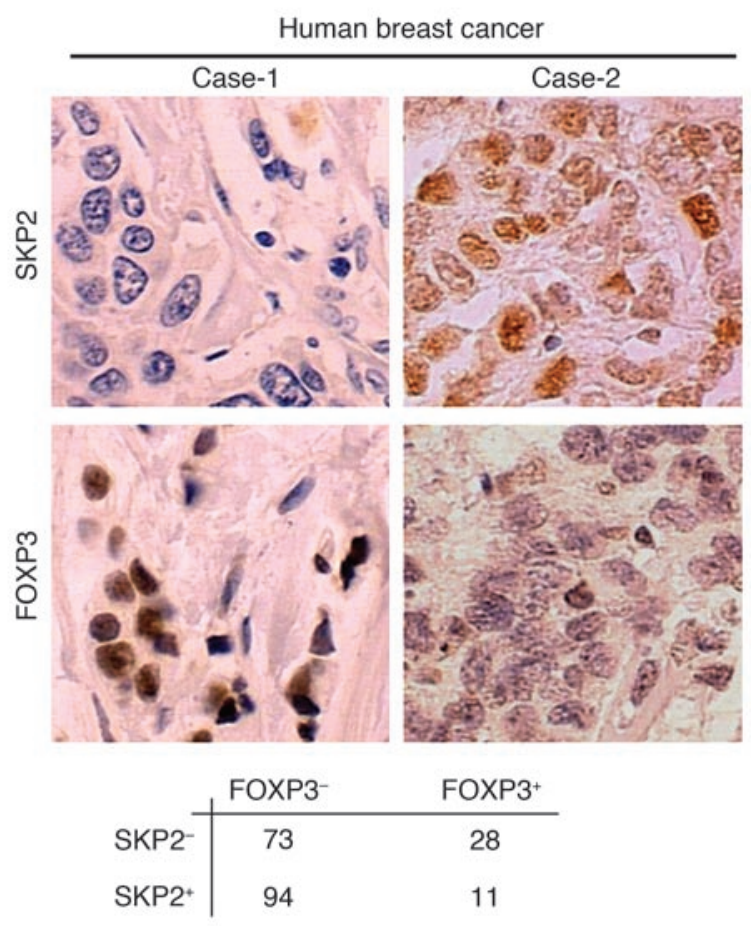

$\chi^{2}$ test: $P=0.0016$

that expressed the mutant allele. More importantly, the spontaneous mammary cancer that developed in the Foxp3 mutant mice, which had completely inactivated the Foxp3 locus, showed drastically higher levels of $S k p 2$ transcripts. This is further supported by the fact that mammary cancers observed in WT BALB/c mice showed much less Skp2 overexpression.

Second, transfection of Foxp3 into mammary cancer cell lines repressed the levels of $S k p 2$ mRNA. Using an $S k p 2$ promoter-driven luciferase reporter, we demonstrated that Foxp3 significantly repressed the promoter activity. Moreover, ChIP assay demonstrated that Foxp3 binds to specific regions of Skp2. The significance of the binding sites was demonstrated by the fact that deletion of either site ablated the repression.

Third, transfection of Foxp3 resulted in an increase of p27, the major target of Skp2. The increase of p27 was likely restricted to the G2/M phase, as a high proportion of Foxp3-expressing cells showed polyploidy, which was also observed in the hepatocytes of

\section{Figure 8}

Ectopic expression of Skp2 substantially alleviates growth inhibition of MCF-7 cell lines by FOXP3. MCF-7 cell lines with inducible expression of either GFP (iii, iv) or FOXP3 (i, ii) were supertransfected with either vector control (i, iii) or SKP2 (ii, iv). After removing untransfected cells by drug selection, the cultures were maintained in tetracycline-free conditions for 2 weeks. Upper and middle panels show photographs of viable (ii, iii, iv) or apoptotic MCF-7 cells. Original magnification, $\times 10$. The lower panel shows colony numbers per $100-\mathrm{mm}^{2}$ plate. At the end of 2 weeks of culture, the dead cells were removed and the plates were stained with violet crystal. The visible colonies were counted. Data shown are means of SD of triplicates and are representative of 3 independent experiments. ${ }^{* \star} P<0.001$ when group I and II were compared by Student's $t$ test.

\section{Figure 7}

Significant reduction in the rate of SKP2 upregulation in the FOXP3+ breast cancer samples. Tissue microarray samples of human breast cancer were stained with either anti-FOXP3 antibody or anti-SKP2 antibody. The samples were scored in a double-blind fashion. The top panels show staining patterns of either FOXP3 or SKP2 in 2 representative cases. Original magnification, $\times 60$. Summary data from 206 independent cases are presented in the lower panel. The $P$ values of the $\chi^{2}$ tests are listed.

the Skp2-deficient mice $(8,9)$. These results indicate that Foxp3 serves as an important regulator for cell cycle progression.

Last, we have demonstrated that the Foxp3-mediated repression of SKP2 occurred in normal and malignant breast epithelial cells in humans. Thus, silencing the FOXP3 gene by siRNA in primary mammary epithelial cells resulted in a significant increase of the SKP2 transcript, while the induced expression of FOXP3 in breast cancer cell lines caused a time-dependent repression of SKP2 expression. More importantly, our analysis of 206 clinical samples demonstrated a substantially reduced rate of SKP2 overexpression among the FOXP3 $3^{+}$breast cancer samples. These data indicate that FOXP3-mediated repression likely serves as an important guardian for overexpression of SKP2 in vivo. It should be noted that, as is often observed among clinical samples, there is not a 1:1 correlation between FOXP3 expression and the absence of SKP2 in breast cancer. Likewise, not all FOXP3- cancers overexpress SKP2. Thus, in addition to FOXP3, other factors also contribute to SKP2 expression. It is worth noting that in non-small cell lung carcinomas, overexpression of Skp2 is associated with the amplification of the Skp2 gene (18). Additional studies are needed to determine whether FOXP3 can attenuate SKP2 expression in the case of gene amplification, as we have demonstrated in the HER2 gene amplification (7).

Taken together, genetic, biochemical, and functional analysis provides strong evidence that Foxp3 is a transcriptional repressor of

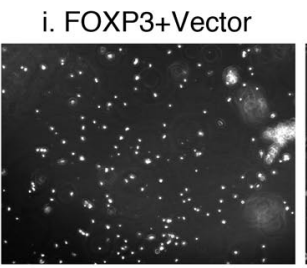

ii. FOXP3+SKP2

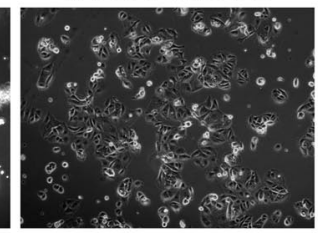

iii. GFP+Vector

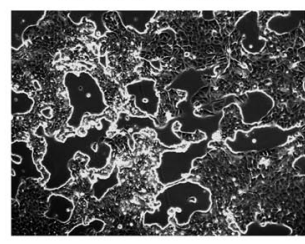

iv. GFP+SKP2
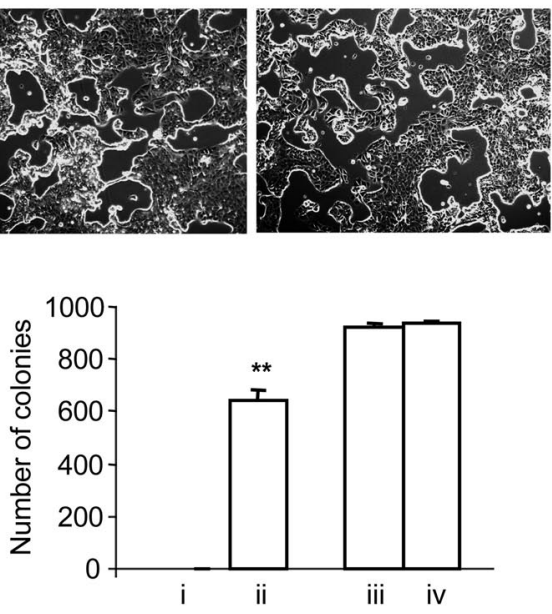
the SKP2 oncogene. Identification of a functionally important nonHer2 target explains why Foxp3 also represses the growth of MCF-7, a breast cancer cell line without HER2/ERBB2 overexpression.

\section{Methods}

Cell lines, experimental animals, and human tissue microarrays. HMECs (HMEC-1; CC-2551) and mammary epithelial cell growth medium (MEGM; CC-3051) were purchased from Cambrex Clonetics. TSA is an aggressive and poorly immunogenic cell line established from the first in vivo transplant of a moderately differentiated mammary adenocarcinoma that arose spontaneously in a BALB/c mouse (31). Breast cancer cell line MCF-7 was purchased from ATCC.

The female mice heterozygous for the Scurfy mutations (Foxp $3^{s / /+}$ ) were backcrossed to the BALB/c background for at least 12 generations at the University of North Carolina (Chapel Hill, North Carolina, USA). The animals were housed at the University Laboratory Animal Facility at The Ohio State University and the University of Michigan under specific pathogen-free conditions. Spontaneous tumor and carcinogen-induced tumor incidence observation were reviewed and approved by The Ohio State University and University of Michigan Institutional Animal Care and Use Committees.

The normal and cancerous breast tissues were obtained from The Ohio State University Department of Pathology Tissue Bank, Zymed Laboratories Inc., and US Biomax Inc.

Antibodies. Affinity purified rabbit anti-Foxp3 antibodies, specific for the FOXP3 N terminus peptide starting at position 25 ([C]LLGTRGS GGPFQGRDLRSGAH), have been described (28) as well as antibodies for immunohistochemical staining and Western blotting (p27 [M-197, 1:500, Santa Cruz Biotechnology Inc.], SKP2 [H-435, 1:100, Santa Cruz Biotechnology Inc.]).

FOXP3-silencing lentiviral vector. The lentivirus-based siRNA-expressing vectors were created by introducing the murine U6 RNA polymerase III promoter and a murine phosphoglycerate kinase promoter-driven (pGK) EGFP expression cassette into a vector of pLenti6/V5-D-TOPO backbone without CMV promoter. A hairpin siRNA sequence of FOXP3 (target sequence at the region of 1256 to 1274 nucleotides; 5'-GCAGCGGACACTCAATGAG-3') was cloned into the lentiviral siRNA-expressing vectors by restriction sites of $A p a \mathrm{I}$ and EcoRI.

ChIP. ChIP was carried out according to a published procedure (32). In brief, the Foxp3-V5-transfected TSA cells were sonicated and fixed with $1 \%$ paraformaldehyde. The anti-V5 antibodies or control mouse IgG were used to pull down chromatin associated with Foxp3-V5. The amounts of the specific DNA fragment were quantitated by real-time PCR and normalized against the genomic DNA preparation from the same cells.

The following are the ChIP real-time PCR primers from upstream Skp2: ChIP-1 forward, TGTGATGGGCACACATACAG; reverse, TGTTCTCT-
GGAAGCCTCAGC; ChIP-2 forward, CGAATCTTGCTCTCTCCACA; reverse, CATGCAAAATTCAGGTGTGC; ChIP-3 forward, GGACAGGCTGTGGATTGAGT; reverse, CCAAGAGGAGCGATGGTTTA; and ChIP-4 forward, TGCTGGGACTTTTCTCCACT; reverse, AGACACCCATGCCTGATAGC; Skp2 forward, TTAGTCGGGAGAACTTTCCAGGTG; Skp2 reverse, AGTCACGTCTGGGTGCAGATTT.

Immunohistochemistry. Expression of Foxp3 and Skp2 in mouse and human breast cancer samples was determined using immunohistochemistry as described. The samples consisting of more than $5 \%$ cells with nuclear expression of Foxp3 were scored as positive. SKP2 scoring followed the previous description (12-15): those with more than $5 \%$ of either nuclear-postive or cytoplasm-positive staining accumulations were scored as Skp2+. FOXP3 and SKP2 staining were scored double blinded.

Colony formation assay. The colony formation assay was carried out as we have described previously (7).

Antibodies, immunoprecipitation, and Western blot. Antibodies specific for SKP2 (Santa Cruz Biotechnology Inc.) and p27 (Cell Signaling) were used for immunoprecipitation and/or Western blot. A special procedure was used to detect polyubiquitinated p27, according to what was reported by Ji et al. (33), using HRP-conjugated ubiquitin (Ub P4D1) (sc-8017, Santa Cruz Biotechnology Inc.).

Statistics. Comparisons between 2 groups were performed using unpaired 2 -tailed Student's $t$ test. ANOVA test was used for analysis of variance between several groups. $\chi^{2}$ test was used for analysis. All values are presented as mean \pm SD. Differences between groups were considered statistically significant at $P<0.05$.

\section{Acknowledgments}

We thank Tim Huang for support and Lynde Shaw and Todd Brown for secretarial assistance. This study is supported by grants from the NIH and the Department of Defense.

Received for publication April 30, 2007, and accepted in revised form September 12, 2007.

Address correspondence to: Y. Liu or P. Zheng, BSRB 2052, 109 Zina Pitcher Place, Ann Arbor, Michigan 48109, USA. Phone: (734) 615-3158; Fax: (734) 763-2162; E-mail: yangl@umich.edu (Y. Liu); panz@umich.edu (P. Zheng).

Tao Zuo and Runhua Liu contributed equally to this work.

Tao Zuo's present address is: Department of Molecular Virology, Immunology, and Molecular Genetics, The Ohio State University, Columbus, Ohio, USA.
1. Vogelstein, B., and Kinzler, K.W. 2002. The genetic basis of human cancer. McGraw-Hill. New York, New York, USA/London, United Kingdom. 821 pp.

2. Macdonald, F., Ford, C.H.J., and Casson, A.G. 2004. Molecular biology of cancer. BIOS Scientific Publishers. London, United Kingdom/New York, New York, USA. 269 pP.

3. Huang, H., et al. 2005. Skp2 inhibits FOXO1 in tumor suppression through ubiquitin-mediated degradation. Proc. Natl. Acad. Sci. U. S. A. 102:1649-1654.

4. Pagano, M. 2004. Control of DNA synthesis and mitosis by the Skp2-p27-Cdk1/2 axis. Mol. Cell. 14:414-416.

5. Nakayama, K.I., and Nakayama, K. 2006. Ubiquitin ligases: cell-cycle control and cancer. Nat. Rev. Cancer. 6:369-381.

6. Schmidt, M., et al. 2002. Cell cycle inhibition by
FoxO forkhead transcription factors involves downregulation of cyclin D. Mol. Cell. Biol. 22:7842-7852.

7. Zuo, T., et al. 2007. FOXP3 is an X-linked breast cancer suppressor gene and an important repressor of HER-2/ErbB2 oncogene. Cell. 129:1275-1286.

8. Nakayama, K., et al. 2000. Targeted disruption of Skp2 results in accumulation of cyclin E and p27(Kip1), polyploidy and centrosome overduplication. EMBO J. 19:2069-2081.

9. Nakayama, K., et al. 2004. Skp2-mediated degradation of $\mathrm{p} 27$ regulates progression into mitosis. Dev. Cell. 6:661-672.

10. Hara, T., et al. 2001. Degradation of p27(Kip1) at the $\mathrm{G}(0)-\mathrm{G}(1)$ transition mediated by a Skp2independent ubiquitination pathway. J. Biol. Chem. 276:48937-48943.

11. Carrano, A.C., Eytan, E., Hershko, A., and Pagano, M. 1999. SKP2 is required for ubiquitin-mediated degradation of the CDK inhibitor p27. Nat. Cell Biol. 1:193-199.

12. Traub, F., Mengel, M., Luck, H.J., Kreipe, H.H., and von Wasielewski, R. 2006. Prognostic impact of Skp2 and p27 in human breast cancer. Breast Cancer Res Treat. 99:185-191.

13. Sonoda, H., et al. 2006. Significance of skp2 expression in primary breast cancer. Clin. Cancer Res. 12:1215-1220.

14. Radke, S., Pirkmaier, A., and Germain, D. 2005. Differential expression of the F-box proteins Skp2 and Skp2B in breast cancer. Oncogene. 24:3448-3458.

15. Signoretti, S., et al. 2002. Oncogenic role of the ubiquitin ligase subunit $\mathrm{Skp} 2$ in human breast cancer. J. Clin. Invest. 110:633-641. doi:10.1172/ JCI200215795.

16. Latres, E., et al. 2001. Role of the F-box protein Skp2 in lymphomagenesis. Proc. Natl. Acad. Sci. 
U. S. A. 98:2515-2520.

17. Shim, E.H., et al. 2003. Expression of the F-box protein SKP2 induces hyperplasia, dysplasia, and low-grade carcinoma in the mouse prostate. Cancer Res. 63:1583-1588.

18. Zhu, C.Q., et al. 2004. Skp2 gene copy number aberrations are common in non-small cell lung carcinoma, and its overexpression in tumors with ras mutation is a poor prognostic marker. Clin. Cancer Res. 10:1984-1991.

19. Zhang, L., and Wang, C. 2006. F-box protein Skp2: a novel transcriptional target of E2F. Oncogene. 25:2615-2627.

20. Imaki, H., et al. 2003. Cell cycle-dependent regulation of the Skp2 promoter by GA-binding protein. Cancer Res. 63:4607-4613.

21. Chatila, T.A., et al. 2000. JM2, encoding a fork head-related protein, is mutated in X-linked autoimmunity-allergic disregulation syndrome. J. Clin. Invest. 106:R75-R81.

22. Brunkow, M.E., et al. 2001. Disruption of a new forkhead/winged-helix protein, scurfin, results in the fatal lymphoproliferative disorder of the scurfy mouse. Nat. Genet. 27:68-73.

23. Bennett, C.L., et al. 2001. The immune dysregulation, polyendocrinopathy, enteropathy, X-linked syndrome (IPEX) is caused by mutations of FOXP3. Nat. Genet. 27:20-21.

24. Wildin, R.S., et al. 2001. X-linked neonatal diabetes mellitus, enteropathy and endocrinopathy syndrome is the human equivalent of mouse scurfy. Nat. Genet. 27:18-20.

25. Lin, W., et al. 2007. Regulatory T cell development in the absence of functional Foxp3. Nat. Immunol. 8:359-368.

26. Fontenot, J.D., Gavin, M.A., and Rudensky, A.Y. 2003. Foxp3 programs the development and function of CD4+CD25+ regulatory T cells. Nat. Immunol. 4:330-336.

27. Fontenot, J.D., et al. 2005. Regulatory T cell lineage specification by the forkhead transcription factor foxp3. Immunity. 22:329-341.
28. Chang, X., et al. 2005. The Scurfy mutation of FoxP3 in the thymus stroma leads to defective thymopoiesis. J. Exp. Med. 202:1141-1151.

29. Marson, A., et al. 2007. Foxp3 occupancy and regulation of key target genes during T-cell stimulation. Nature. 445:931-935.

30. Zheng, Y., et al. 2007. Genome-wide analysis of Foxp3 target genes in developing and mature regulatory T cells. Nature. 445:936-940.

31. Giovarelli, M., et al. 1995. Local release of IL-10 by transfected mouse mammary adenocarcinoma cells does not suppress but enhances antitumor reaction and elicits a strong cytotoxic lymphocyte and antibody-dependent immune memory. J. Immunol. 155:3112-3123.

32. Im, H., et al. 2004. Measurement of proteinDNA interactions in vivo by chromatin immunoprecipitation. Methods Mol. Biol. 284:129-146.

33. Ji, P., et al. 2004. An Rb-Skp2-p27 pathway mediates acute cell cycle inhibition by $\mathrm{Rb}$ and is retained in a partial-penetrance Rb mutant. Mol. Cell. 16:47-58. 